\title{
Paciente con regurgitación: cómo estudiarla y cuáles son sus principales diagnósticos diferenciales
}

\author{
Mariela Dulbecco, Yolanda Vargas Rodríguez, Mauricio Guzmán \\ Unidad de Neurogastroenterología, Servicio de Gastroenterología, Hospital Interzonal General de Agudos General José de San Martín. Universidad \\ Nacional de La Plata. Buenos Aires, Argentina.
}

Acta Gastroenterol Latinoam 2021;51(1):29-38

Recibido: 04/03/2021 / Aceptado: 08/03/2021 / Publicado online: 22/03/2021 / https://doi.org/10.52787/jagj4273

\begin{abstract}
Resumen
La enfermedad por reflujo gastroesofágico es uno de los diagnósticos más frecuentes realizados en la práctica gastroenterológica. En esta revisión, se plantearán los potenciales confundidores del reflujo gastroesofágico. Según la definición de Montreal, "es una condición que se desarrolla cuando el reflujo del contenido gástrico causa sintomas molestos y / o complicaciones". Sin embargo, es cada vez más claro que, a veces, los sintomas que sospechamos que son causados por la enfermedad por reflujo gastroesofágico constituyen la expresión de otros trastornos funcionales, trastornos conductuales e, inclusive, lesiones estructurales. De esta complejidad, surgen los confundidores del reflujo, en donde la rumiación y los eructos supragástricos pueden presentarse con sintomas similares, ser tratados inicialmente con pautas basadas en el uso de los inhibidores de la bomba de protones y asi ser erróneamente enfocados. De la misma manera, la regurgitación puede ser la expresión sintomática de diferentes desórdenes funcionales y no, exclusivamente, un sintoma "típico" de la enfermedad por reflujo gastroesofágico. De aqui la necesidad
\end{abstract}

Correspondencia: Mauricio Guzmán

Correo electrónico: mauricioguzman76@hotmail.com y el desafio para el médico tratante de identificar correctamente los mecanismos fisiopatológicos responsables de los sintomas del paciente para un correcto abordaje terapéutico.

Palabras claves. Enfermedad por reflujo gastroesofágico, regurgitación, rumiación, eructos supragástricos.

\section{Patient with Regurgitation: How to Study It and Its Main Differential Diagnoses}

\section{Summary}

In gastroenterological practice, gastroesophageal reflux disease is one of the most frequent diagnoses. In this article the potential confounding of gastroesophageal reflux will be raised. According to the Montreal definition, "is a condition that develops when the reflux of stomach contents into the esophagus causes troublesome symptoms and/or complications. "However, it is becoming increasingly clear that sometimes symptoms suspected to be caused by gastroesophageal reflux disease are the expression of other functional and behavioral disorders or even structural lesions. From this complexity arise reflux confounding, where rumination and supragastric belching may present symptoms similar to gastroesophageal reflux disease, be initially treated with the proton pump inhibitor-based guidelines, and thus be mistakenly targeted. Likewise, regurgitation may be the symptomatic expression of different functional disorders and not exclusively a "typical" symptom of gastroesophageal reflux disease. Hence the need and the challenge for the treating physician to correctly identify the pathophysiological mech- 
anisms responsible for the patient's symptoms for a correct therapeutic approach.

Keywords. Gastroesophageal reflux disease, regurgitation, rumination, supragastric belching.

\section{Abreviaturas}

ERGE: Enfermedad por reflujo gastroesofágico.

IBP: Inhibidores de la bomba de protones.

ERNE: Enfermedad por reflujo no erosiva.

TEA: Tiempo de exposición ácida.

IS: Índice sintomático.

HRIM: Manometría esofágica de alta resolución con impedanciometría.

EEI: Esfinter esofágico inferior.

EES: Esfinter esofágico superior.

MII-pH: Multichannel intraluminal impedance monitoring combined with $p H$-metry (Monitoreo ambulatorio multicanal de impedancio-pH-metría).

EMG: Electromiografía.

PAS: Probabilidad de asociación sintomática.

DMS-5: Manual de desórdenes mentales, $5^{\text {ta }}$ edición.

\section{Introducción}

La enfermedad por reflujo gastroesofágico (ERGE) es uno de los diagnósticos más comunes realizados en la práctica gastroenterológica. Se la define, de acuerdo con el consenso de Montreal, "como una condición que se desarrolla cuando el reflujo del contenido gástrico causa síntomas molestos y/o complicaciones". Los síntomas típicos de la ERGE incluyen la pirosis y la regurgitación (síndrome de reflujo típico). ${ }^{1}$ La ERGE se considera una afección común que afecta hasta el $20 \%$ de la población en Occidente. ${ }^{2}$ Un estudio poblacional realizado en Argentina identificó una prevalencia de los síntomas frecuentes del reflujo gastroesofágico del 23\% (al menos una vez por semana) y definió la prevalencia de la ERGE en un $11,9 \%$ cuando los síntomas se presentan al menos 2 veces por semana. ${ }^{3}$ Sin embargo, los síntomas típicos (pirosis y regurgitación) mostraron en una revisión sistemática una subóptima sensibilidad (30-76\%) y especificidad (62-96\%) para el diagnóstico de la ERGE. ${ }^{4}$

Actualmente, de acuerdo con el consenso de Lyon, también podemos definir a la ERGE según las complicaciones halladas en la endoscopía como ser la esofagitis erosiva grados C y D de la clasificación de Los Ángeles, la presencia del esófago de Barrett (mayor de $1 \mathrm{~cm}$ ), o ante el hallazgo de una estenosis péptica. ${ }^{5}$ Un estudio en nuestro medio determinó que estas complicaciones únicamente están presentes en el $40 \%$ de los pacientes con síntomas típicos de la ERGE. Así mismo, se determinó que en el 60\% de los casos la ERGE no genera daño en la mucosa esofágica evidente por endoscopía, lo que denominamos: enfermedad por reflujo no erosiva (ERNE). ${ }^{6}$ En este grupo de pacientes, el diagnóstico definitivo requiere ser confirmado por los métodos funcionales, como el monitoreo ambulatorio del reflujo mediante $\mathrm{pH}$-metría o $\mathrm{pH}$-impedanciometría. ${ }^{5}$ Una caída del $\mathrm{pH}$ esofágico por debajo de 4 es el umbral que mejor discrimina un episodio de reflujo. La suma del tiempo en el cual el $\mathrm{pH}$ se encuentra por debajo de este umbral se utiliza para determinar el tiempo de exposición ácida (TEA), el cual se expresa en porcentaje y se considera anormal cuando es mayor al 6\%. ${ }^{5}$

La asociación positiva o negativa entre los síntomas del paciente y los episodios de reflujo, medidos por el índice sintomático (IS) y la probabilidad de asociación sintomática (PAS), es la relación que existe entre los episodios de reflujo evidenciados por la impedanciometría y la presencia de los síntomas registrados por el paciente. Cuando existe un TEA normal (menor al 4\%) y la asociación sintomática es positiva, se va a denominar: hipersensibilidad al reflujo. En cambio, si la asociación sintomática es negativa, se llamará: pirosis funcional. Tanto la hipersensibilidad al reflujo como la pirosis funcional, son cuadros funcionales del esófago definidos por el consenso de Roma IV.

El abordaje terapéutico de los pacientes con la ERGE incluye medidas higiénico dietéticas, cambios en el estilo de vida y fármacos bloqueantes de la secreción ácida. De estos últimos, los IBP se consideran por sobre el resto dado su mejor eficacia para el alivio sintomático del paciente. La respuesta a la administración empírica de los IBP es una alternativa terapéutica razonable para confirmar la ERGE, aunque un metaanálisis confirmó algunas limitaciones de este abordaje, mostrando una sensibilidad del $78 \%$ y una especificidad del $54 \% .^{7}$ Aproximadamente entre un 10 y un $40 \%$ de los pacientes con sospecha de ERGE no responden al tratamiento con los IBP. La tasa de respuesta a los IBP en los pacientes varía también en función del síntoma predominante. Si el paciente se presenta con pirosis, la tasa de respuesta sintomática a los IBP (con o sin esofagitis acompañante) es del 56\%, mientras que esa respuesta disminuye al $47 \%$ ante la presencia de regurgitación. Esto se explica porque los mecanismos implicados en el desarrollo del síntoma predominante son distintos. ${ }^{8-10}$

Esta población de pacientes representa una gran carga para el sistema de salud y se necesitan estrategias para caracterizar y manejar de forma efectiva este grupo tan 
heterogéneo. Algunos estudios informan que aproximadamente un tercio de los pacientes remitidos por falta de respuesta a los IBP sufre trastornos gastroduodenales funcionales, incluido el síndrome de rumiación y los eructos supragástricos. ${ }^{8,9}$ Por lo tanto, no todos los pacientes se verán beneficiados de una mayor potencia de la inhibición del ácido y muchos se pueden ver potencialmente perjudicados ante la intervención quirúrgica de la ERGE. ${ }^{8}$

Por todo lo expuesto, se considera muy importante diferenciar fenotipos de la ERGE, ya que cada uno tiene un enfoque terapéutico distinto (la ERGE, la hipersensibilidad al reflujo y la pirosis funcional). Existen ciertos confundidores que pueden desviar el abordaje diagnóstico y, por lo tanto, la respuesta a las intervenciones terapéuticas. Por eso, ante la presencia de un paciente que no responde al tratamiento con los IBP, es de suma importancia identificar mecanismos adicionales que se vinculen con esta refractariedad y simulen o se confundan en su expresión con los síntomas típicos de la ERGE. ${ }^{10}$

En los últimos años, ha cobrado un gran interés el diagnóstico de los eructos supragástricos y el síndrome de rumiación, siendo estos desórdenes conductuales los causales de los síntomas que se interpretan como un reflujo refractario. Existen elementos clínicos y métodos complementarios, mediante estudios funcionales, que nos permiten arribar a un correcto diagnóstico. Asimismo, se deben tener en cuenta, inicialmente, las alteraciones estructurales y, luego, descartar los trastornos motores esofágicos que suelen expresarse con síntomas similares a los de la ERGE, principalmente la regurgitación, y que en la evaluación de los pacientes no pueden ser pasados por alto. ${ }^{10,11}$

El objetivo de esta presentación es ofrecer una revisión acerca de los confundidores más prevalentes en la ERGE para facilitar su identificación clínica y su correcto manejo.

\section{Regurgitación}

Se define a la regurgitación, en el contexto de la ERGE, como la percepción del retorno repentino, sin esfuerzo y no precedido de eructos, del contenido gástrico hacia la boca o la hipofaringe e implica la relajación o insuficiencia cricofaringea. ${ }^{1}$ La ERGE es una causa frecuente de regurgitación, aunque no la única. Mientras la ERGE es encontrada en el $56 \%$ de los pacientes con pirosis predominante, solamente el $29 \%$ de los que presentan regurgitación predominante la confirma. ${ }^{12}$ Asimismo, la regurgitación posprandial persistente puede ser encontrada en hasta el $40 \%$ de los pacientes con la ERGE refractaria al tratamiento con los IBP. ${ }^{13} \mathrm{La}$ ganancia terapéutica de los IBP, en comparación con un placebo, en el alivio de la regurgitación es un 20\% menor que para la pirosis. ${ }^{14}$

Esto probablemente se deba a una superposición significativa entre la ERGE y los trastornos funcionales, los trastornos de la conducta, las lesiones estructurales o los desórdenes de la alimentación que pueden presentarse con episodios recurrentes de regurgitación.

Las lesiones estructurales que obstruyan la luz esofágica pueden generar regurgitación. Así mismo, también puede aparecer en los trastornos motores esofágicos como la acalasia. Los pacientes que presentan como síntoma predominante la regurgitación de líquidos o alimentos tienen una probabilidad pretest más alta de dismotilidad esofágica y de síndrome de rumiación que de la ERGE. ${ }^{15}$

El vómito puede a veces confundirse con la regurgitación e, inclusive, puede estar presente en el mismo paciente. Cuando este refiere vómitos, la ausencia de arcadas, náuseas y contracciones abdominales, estás pueden ser de utilidad para diferenciarlo de la regurgitación. ${ }^{16}$

El confundidor más relevante a la hora de evaluar un paciente con la ERGE con regurgitación predominante es el síndrome de rumiación. Su correcta identificación evitaría un desenlace negativo en los pacientes a quienes se les indica una cirugía tras confirmar la ERGE, ya que este síndrome puede ser la causa de la falta de respuesta al tratamiento con los IBP y suele mejorar con el tratamiento con terapia cognitiva conductual y no con la cirugía antirreflujo.

Por lo tanto, la evaluación inicial debe incluir la derivación temprana para una manometría esofágica de alta resolución con impedanciometría (HRIM) después de que una endoscopia haya descartado una obstrucción mecánica, la esofagitis erosiva o una gran hernia de hiato. El protocolo de manometría puede modificarse para incluir un período posprandial de evaluación si el paciente describe regurgitación relacionada con las comidas y existe la sospecha del síndrome de rumiación. ${ }^{15}$

\section{Eructos}

El eructo se define como "la expulsión oral del aire desde el tracto gastrointestinal superior que puede ser audible o en algunos casos ocurrir de forma silenciosa". ${ }^{17}$ Si bien todos los sujetos sanos presentan eructos, se los considera molestos cuando se convierten en excesivos o desencadenan síntomas de reflujo. ${ }^{17}$

La ERGE es la condición más relevante para los dos tipos de eructos. La importancia en la práctica clínica radica en identificar los eructos supragástricos, ya que (como desarrollaremos más adelante) son los que constituyen una potencial causa de refractariedad al tratamiento y presentan un enfoque terapéutico diferente. ${ }^{17,18}$ 
Estos pacientes pueden acudir a la consulta por el eructo en sí (fenómeno audible), mientras que en otros puede manifestarse únicamente con síntomas típicos de reflujo. ${ }^{18}$

\section{- Epidemiología}

Múltiples estudios informan la prevalencia de los eructos en la población general con valores que van desde el $6,7 \%$ al $28,8 \%{ }^{19,20}$

Los eructos son más frecuentes en los pacientes que tienen síntomas típicos concomitantes de reflujo, aunque su prevalencia en los pacientes con ERGE también es muy variable, con rangos entre el $4,1 \%$ al $75,6 \%{ }^{21}$ Esta heterogeneidad se debe a que en los estudios incluyen los dos tipos de eructos y a que las definiciones de "eructos excesivos" son variables. Se estima una prevalencia del 3,4\% de los eructos supragástricos en la población con los síntomas gastrointestinales altos. ${ }^{22}$

\section{- Tipos de eructos: gástricos y supragástricosa}

Una correcta historia clínica a menudo nos puede orientar en la identificación del tipo de eructo. No obstante, la monitorización por impedanciometría de $24 \mathrm{~h}$ es la modalidad diagnóstica de elección, ya que proporciona la evidencia objetiva del sentido del movimiento del gas en el esófago, así como su eventual relación con el reflujo y los síntomas. ${ }^{23}$

\section{- Eructos gástricos}

Con cada deglución, ingerimos un volumen variable de aire que es transportado al estómago. Los eructos gástricos son un fenómeno fisiológico y constituyen el mecanismo que previene la acumulación excesiva de gas en el estómago y en el duodeno. Este fenómeno ocurre en casi todos los individuos, de manera no audible, involuntaria, a una tasa promedio de 30 episodios/24 horas. ${ }^{17,24,25}$

El mecanismo responde con la distensión del estómago proximal por el aire, que desencadena las relajaciones transitorias del esfínter esofágico inferior (EEI), que permiten que el aire sea liberado de forma retrograda a la faringe. Las relajaciones transitorias del EEI son un reflejo vagal vía el tronco encefálico, no un reflejo local. ${ }^{24,25}$

Los eructos gástricos se pueden reconocer con la monitorización de la impedanciometría como un aumento del nivel de impedancia que comienza en el canal distal y progresa hasta el más proximal ${ }^{24}$ (Figura $1 \mathrm{~A}$ ).

$\mathrm{La}$ asociación entre los eructos gástricos y la ERGE debe ser aún elucidada. Si bien los monitoreos de

Figura 1. Trazado de la impedanciometría: configuración típica de los subtipos de eructos. A la izquierda puede verse el eructo gástrico (A); a la derecha, el eructo supragástrico (B). Gentileza del sector de Neurogastroenterología del Hospital Interzonal General de Agudos General José de San Martín de La Plata.

Eructo gástrico

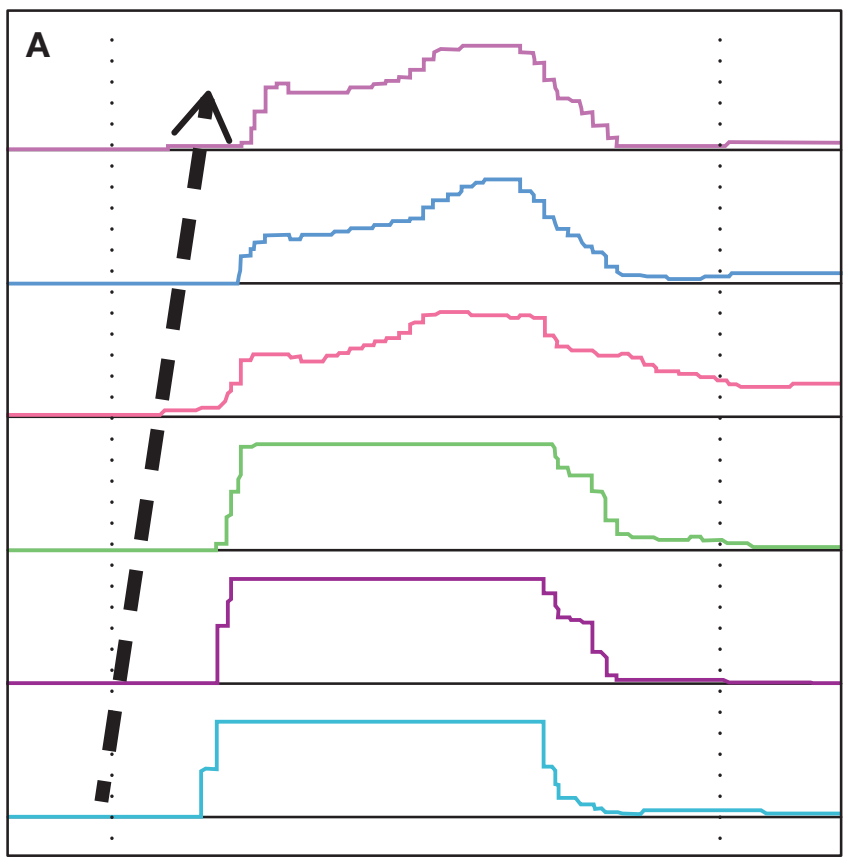

Eructo supragástrico

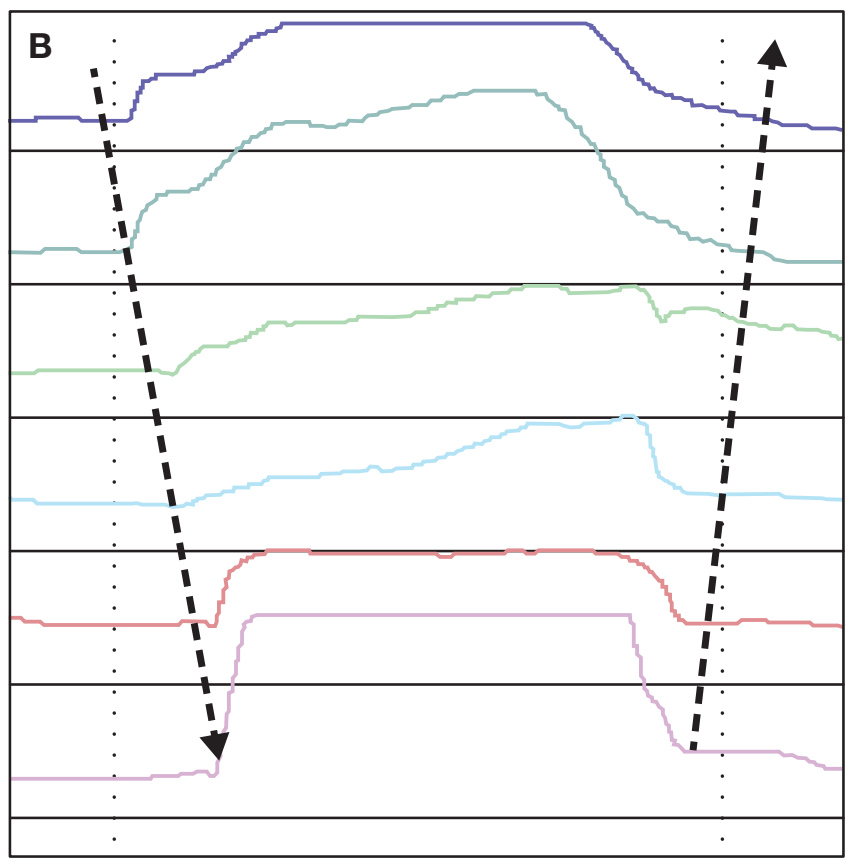


pH-impedanciometría de 24 horas muestran que los pacientes con la ERGE presentan mayor aerofagia y eructos gástricos que los pacientes normales, no se ha podido establecer que estos eventos fuesen la causa de los episodios de reflujo. ${ }^{18,26}$

\section{- Eructos supragástricos}

A diferencia de los eructos gástricos, los supragástricos se consideran un trastorno de la conducta. El paciente succiona el aire, que rápidamente es llevado al esófago y es seguido de su rápida expulsión. Esto se puede demostrar fácilmente con el monitoreo de la impedanciometría, en donde se evidencia que el aire no se origina ni alcanza al estómago. ${ }^{17,24,25}$ Los eructos supragástricos se identifican como un aumento en la impedancia, comenzando en el canal proximal y progresando hacia un canal distal, seguido de un retorno a la línea de base que empieza en el canal distal $^{23}$ (Figura 1B).

Se cree que este trastorno podría comenzar como una respuesta voluntaria a alguna molestia o incomodidad en la garganta, el tórax o el abdomen. Los pacientes aprenden este comportamiento para tratar de aliviar esa sensación y, con el tiempo, se convierte en un acto inconsciente y repetitivo. ${ }^{24}$ En algunos casos, los pacientes refieren el momento en que comenzaron a tener eructos, por lo que se postula que los eventos estresantes de la vida pueden precipitar los eructos supragástricos. ${ }^{18}$

Existen dos mecanismos para generar estos eructos: $:^{24,25}$

- El primero (y el más común) es el denominado método de succión. Se caracteriza por un movimiento del diafragma en la dirección aboral, lo que resulta en una presión intratorácica negativa y una relajación del esfínter esofágico superior (EES) durante la cual la glotis se encuentra cerrada y, por lo tanto, el aire fluye desde la presión atmosférica en la faringe hasta la presión subatmosférica en el esófago. Posteriormente, el aire esofágico se expulsa por vía oral como resultado del esfuerzo y el paciente percibe el eructo.

- El segundo mecanismo se lo define como método de inyección. Está caracterizado por un aumento simultáneo de la presión en la faringe que inicia la entrada del aire en el esófago. En este mecanismo, la fuerza impulsora del flujo de aire es el gradiente de presión entre la presión faríngea elevada y la intraesofágica sin cambios.

\section{- Evaluación clínica}

Cuando el paciente consulta por eructos de forma aislada, casi invariablemente se trate de eructos supragástricos. Una característica distintiva en la historia clínica es que los eructos supragástricos no se producen durante el habla, ni el sueño, y aumentan durante eventos estresantes (los eructos aparecen cuando el médico pregunta y desaparecen cuando el paciente responde). ${ }^{25-27}$ El valor umbral considerado como normal de eructos supragástricos es $\leq 13$ episodios $/ 24 \mathrm{~h} .{ }^{22}$ Sin embargo, se debe tener en cuenta que distintos factores personales pueden alterar el umbral para hacer percibir a los eructos como molestos.

\section{- Eructos supragástricos y su papel como confundi- dores en la ERGE}

La prevalencia de este trastorno de la conducta varía según el fenotipo de la ERGE, evidenciándose una prevalencia del $37,5 \%$ en la enfermedad por reflujo no erosiva (ERNE: TEA > 6\%), del 39,7\% en la hipersensibilidad al reflujo (TEA $<4 \%$ con una asociación sintomática) y del $22 \%$ en los pacientes con pirosis funcional (TEA $<4 \%$ sin una asociación sintomática). Aún más importante es que los eructos supragástricos son potencialmente responsables del $40 \%$ de los síntomas y, por lo tanto, de la falta de respuesta al tratamiento habitual en la hipersensibilidad al reflujo. ${ }^{13}$

Los eructos supragástricos provocan los síntomas típicos de la ERGE (pirosis, regurgitación o dolor de pecho) de dos formas: ${ }^{18}$

1) por la inducción del reflujo propiamente dicho;

2) por la distensión esofágica producida por el aire.

En pacientes con eructos supragástricos excesivos, al menos $1 / 3$ del tiempo de exposición ácida puede ser consecuencia de los eructos. ${ }^{28}$

Se han planteado tres posibles mecanismos por los cuales los eructos supragástricos inducen el episodio de reflujo: ${ }^{18}$

1) la distensión esofágica producida por el eructo supragástrico podría provocar una relajación transitoria del EEI;

2) un aumento en la presión abdominal podría llevar el contenido gástrico a través del EEI;

3) la relajación transitoria del EEI podría inducir un reflejo de relajación del EES, quien en consecuencia permitiría al aire ingresar al esófago.

Por lo expuesto, se entiende la importancia de la pesquisa de los eructos supragástricos en los pacientes refractarios a los IBP, ya que su tratamiento es requerido para mejorar los síntomas en un porcentaje de pacientes con la ERGE refractaria.

\section{- Tratamiento}

El tratamiento de los eructos supragástricos consiste en la terapia de fonación o en la terapia cognitiva conductual. Ambas son eficaces. La elección va a depender de la experiencia y disponibilidad de cada centro. ${ }^{18}$ 
Terapia de fonación: este abordaje evidenció una mejoría en el $83 \%$ de los pacientes. Consiste en unas técnicas llevadas a cabo por fonoaudiólogos con experiencia en trastornos de la voz o la deglución. Se inicia con una explicación del mecanismo de producción de los eructos, la creación de la conciencia de las maniobras de succión o inyección de aire y la realización de ejercicios para mejorar los movimientos del complejo lingual, laríngeo y cricofaríngeo que eviten la apertura del EES cuando no sea necesaria. ${ }^{29}$

Terapia cognitiva conductual: Este abordaje evidenció la disminución objetiva de los episodios de eructos supragástricos en más del $50 \%$. La parte cognitiva de esta terapia consiste en explicarle al paciente el mecanismo de los eructos supragástricos para ayudarlo a comprender el trastorno, su probable inicio y sus eventos desencadenantes, además de proveerle la justificación para realizar los ejercicios. Se debe orientar al paciente para identificar alguna señal de advertencia (sensaciones premonitorias) como pueden ser las molestias en la garganta, el pecho o el abdomen para tener la posibilidad de cortar el inicio de los eructos. La parte conductual se basa en el uso de la respiración diafragmática y la apertura de la boca con la posición de la lengua para que sea físicamente imposible realizar los eructos. ${ }^{29}$

La respiración diafragmática consiste en una inspiración y espiración prolongada que movilicen la pared abdominal y no la pared torácica. Para esto es útil indicarle al paciente que coloque una mano en el tórax y otra en el abdomen logrando que la inspiración prolongada (al menos de 3 segundos) eleve la mano apoyada en el abdomen. Los pacientes deben respirar por la boca con una apertura moderada y colocar la lengua detrás de los dientes frontales superiores. Estos procedimientos deben entrenarse al menos dos veces al día durante 3 a 5 min en decúbito supino o sentado. Una vez que los pacientes se acostumbran a la técnica de respiración, se recomienda usarla con tanta frecuencia como sea posible, especialmente cuando se percibe una señal de advertencia o se inician los eructos. ${ }^{28,30}$

En resumen, se considera fundamental: el correcto diagnóstico del paciente con los síntomas de reflujo, caracterizar el fenotipo de la ERGE y, ante la refractariedad al tratamiento, pesquisar los eructos supragástricos para así enfocar el tratamiento, ya sea con los IBP, los neuromoduladores o la terapia conductual, según lo requiera.

\section{Rumiación}

El síndrome de la rumiación es un desorden funcional gastrointestinal de etiología desconocida caracterizado por la regurgitación hacia la boca, muchas veces repetitiva, de la comida recientemente ingerida en ausencia de anormalidades estructurales. Este fenómeno es considerado un desorden del comportamiento. El material regurgitado puede ser nuevamente deglutido o vomitado por el paciente. Típicamente la regurgitación no es precedida por náuseas o arcadas y no es selectiva para líquidos o sólidos. ${ }^{24,31}$

En los últimos años, este desorden del comportamiento ha cobrado interés por ser uno de los confundidores a tener en cuenta ante la presencia de los pacientes con reflujo refractario con predominio de regurgitación, aunque también debe tenerse en cuenta en los pacientes con pirosis.

La disponibilidad de la manometría esofágica de alta resolución con impedanciometría (HRIM) y la necesidad de considerar a la rumiación dentro de los diagnósticos diferenciales en los pacientes con regurgitación posprandial y reflujo refractario nos exigen un desafío en nuestra práctica clínica.

Si bien las características de los síntomas permiten sospechar el diagnóstico de rumiación, se lo debe confirmar con métodos objetivos, ya que los criterios clínicos tienen un valor predictivo positivo de solo el $50 \% .{ }^{32}$ La HRIM con protocolo posprandial sería el método objetivo que mejor nos permitiría confirmar y clasificar a la rumiación para, de esta manera, otorgar el tratamiento adecuado a cada subtipo. ${ }^{33}$

\section{- Epidemiología y su relación con la ERGE}

La prevalencia real del síndrome de rumiación sigue siendo desconocida, en parte porque los datos son limitados y, por otro lado, por la falta de conocimiento sobre esta entidad por muchos médicos. De acuerdo con la $5 .{ }^{a}$ edición del manual de desórdenes mentales (DMS-5), la prevalencia varía entre un $0,8 \%$ y un $10,6 \%$ en la población. ${ }^{34}$ Un estudio epidemiológico australiano mostró una prevalencia del $0,3 \%$, mientras que otro estudio mexicano arrojó una prevalencia del 0,8\%.35, 36

En los pacientes con síntomas de reflujo refractario al tratamiento con los IBP, la prevalencia de rumiación ha sido poco explorada. Yadlapati et al. sugieren que hasta un $20 \%$ de los pacientes con la ERGE refractaria pueden presentar rumiación posprandial y que esta distinción entre regurgitación y rumiación suele ser muy difícil de aclarar, aunque no menos importante. ${ }^{37}$

Sawada et al. determinaron que el 9,5\% de los pacientes con hipersensibilidad al reflujo que no responden al tratamiento presenta rumiación asociada diagnosticada mediante la HRIM y el monitoreo con $\mathrm{pH}$-impedanciometría de $24 \mathrm{~h} .{ }^{13}$ 


\section{- Diagnósticos diferenciales}

La rumiación debe siempre ser tomada en cuenta dentro de los diagnósticos diferenciales en los pacientes con regurgitación postprandial y vómitos. Una historia clínica detallada debe ser realizada en todo paciente con sospecha de rumiación. Algunas características distintivas suelen diferenciar la rumiación de los vómitos, ya que la primera no suele estar precedida de náuseas, arcadas o esfuerzos. A diferencia de la rumiación, los vómitos en los pacientes con gastroparesia suelen ser más tardíos y su composición no es de comida recién ingerida.

A diferencia de la regurgitación de la ERGE, los episodios de rumiación suelen no tener sabor ácido y es raro que ocurran durante el sueño o en los periodos de ayuno. El uso de los IBP no suele mejorar los síntomas y, por el contrario, puede perpetuarlos al bloquear el sabor ácido que habitualmente lleva a autolimitar los episodios de rumiación. En ocasiones, estos pacientes son catalogados como casos de reflujo refractario en forma errónea. En los pacientes con acalasia, los síntomas suelen ser similares y difíciles de diferenciar, aunque la presencia de la disfagia debe orientar el diagnóstico. En estos casos, una evaluación estructural y funcional debe ser considerada. ${ }^{31}$

\section{- Etiología y fisiopatología}

La fisiopatología del síndrome de la rumiación sigue siendo desconocida. Los episodios de rumiación pueden ser disparados por un aumento en la presión intragástrica producida por una contracción voluntaria, aunque no intencionada, de los músculos de la pared abdominal. Esto puede vencer la resistencia del EEI, favoreciendo el paso del contenido gástrico recientemente ingerido hacia el esófago. Una vez que el material ingresa al cuerpo esofágico, se produce la relajación del esfínter esofágico superior, alcanzando así la orofaringe. En esta instancia, el contenido gástrico regurgitado puede ser deglutido nuevamente o vomitado.

Varios estudios sugieren que el síndrome de rumiación es un desorden del comportamiento desencadenado por un discomfort abdominal, así como una reacción a un evento de estrés. ${ }^{31,38,39}$

El mecanismo por el cual se produce un aumento en la presión intragástrica se puede vincular con los cambios en la actividad muscular abdómino-torácica y esto ha sido demostrado mediante una electromiografía (EMG). Barba et al. proponen que la rumiación es producida por una respuesta somática no percibida a la ingestión de la comida. Mediante la EMG se describe como los episodios de rumiación se producen por una maniobra abdóminotorácica coordinada, que consiste en una contracción repentina de los músculos intercostales con la elevación de la pared costal, a la que llaman succión torácica, junto con una contracción de los músculos abdominales anteriores, denominada compresión abdominal. El diafragma facilita la creación de un fenómeno de cavidad común entre el abdomen y el tórax al relajar el hiato (apertura hiatal) y permitir el desplazamiento hacia arriba del contenido gástrico. Así mismo, se describe la efectividad de la biorretroalimentación guiada por la EMG como una opción potencial de tratamiento para la rumiación. ${ }^{40}$

Las alteraciones en la función gástrica también han sido descriptas en los pacientes con rumiación. Mediante la valoración de la acomodación gástrica con baróstato, se han podido demostrar anormalidades en la misma hasta en el $50 \%$ de los pacientes en comparación con los controles. ${ }^{41}$ Sin embargo, el vaciamiento gástrico parece no verse afectado en estos pacientes y los tratamientos destinados a mejorarlo no han mostrado efectividad en revertir los episodios de rumiación. ${ }^{42}$

\section{- Diagnóstico}

El diagnóstico clínico se basa en los criterios de Roma IV para los desórdenes funcionales digestivos gastroduodenales. ${ }^{43}$ El DMS-5 también provee datos clínicos para el diagnóstico del síndrome de rumiación. ${ }^{34}$

Los episodios de rumiación ocurren típicamente en el periodo postprandial, 10 a 15 minutos después de la ingesta, aunque pueden persistir hasta 1 o $2 \mathrm{~h}$. También pueden ocurrir después de la ingesta de sólidos o líquidos. La regurgitación del material puede ser remasticada y nuevamente deglutida o vomitada. ${ }^{38}$

Los episodios de rumiación han sido demostrados y caracterizados mediante múltiples estudios funcionales digestivos. Actualmente, el método que mejor demuestra estos episodios es la HRIM. Así se puede identificar un aumento en la presión intragástrica que debe ser mayor a $25 \mathrm{mmHg}$, coincidente con el inicio del movimiento retrogrado del contenido gástrico hacia el esófago en la impedanciometría. ${ }^{44,45}$

Bredenoord et al. describen un patrón típico para realizar el diagnostico mediante la HRIM y establecen criterios objetivos (Figura 2). ${ }^{44} \mathrm{~A}$ su vez, este autor establece que los episodios de rumiación pueden ocurrir por 3 mecanismos distintos:

1) rumiación primaria, cuando la rumiación no es precedida por ningún evento;

2) rumiación secundaria, cuando es precedida por un evento de reflujo;

3) y rumiación asociada a un eructo supragástrico, cuando es precedida por un eructo supragástrico.

La característica distintiva de cada uno de los subtipos de rumiación es el aumento en la presión intragástrica que excede los $25 \mathrm{mmHg}$, la cual presenta una especificidad diagnóstica del $100 \%$. $^{31,44}$ 
Figura 2. Episodio de rumiación. Manometría esofágica de alta resolución con impedanciometría. Aumento de la presión gástrica (círculos negros), seguido del movimiento retrogrado del contenido gástrico hacia la hipofaringe (visible en la impedanciometría). Gentileza del sector de Neurogastroenterología del Hospital de Clínicas José de San Martín.

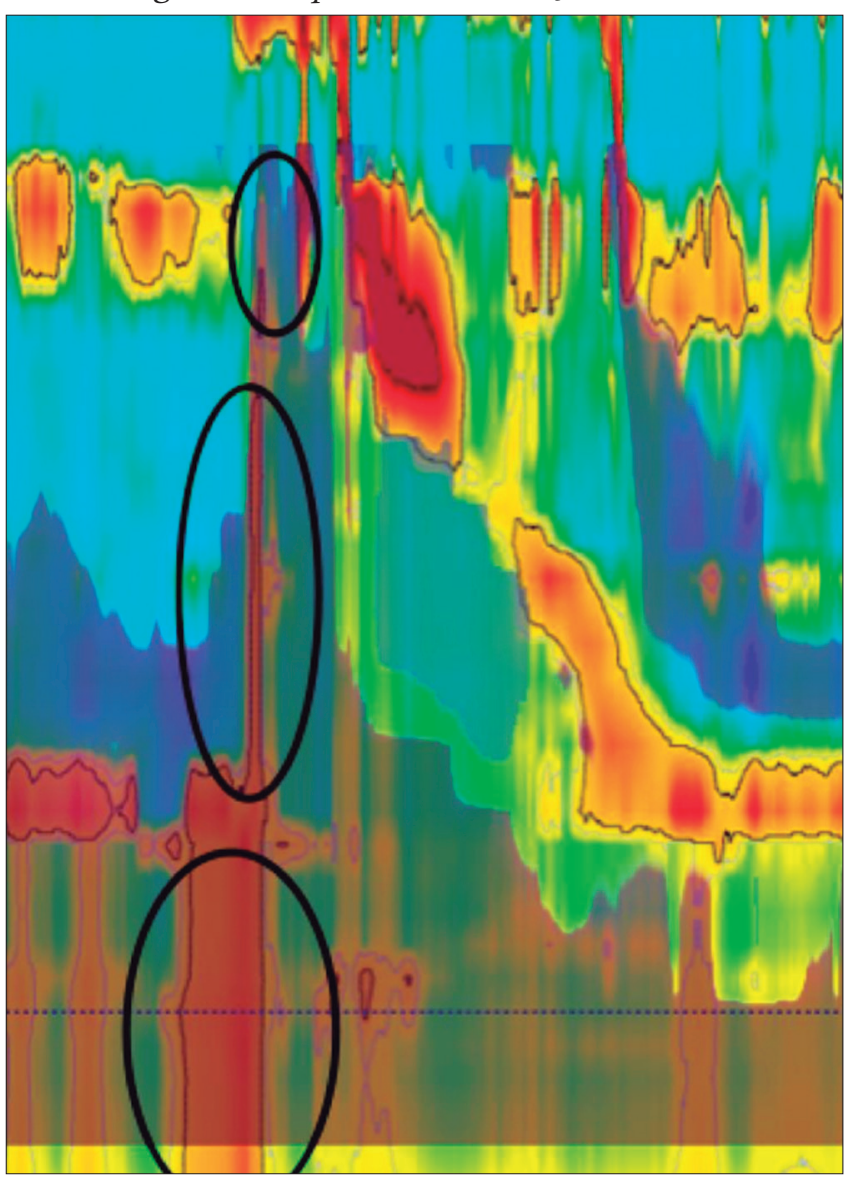

Nakagawa et al. describen un patrón distintivo de rumiación usando un monitoreo ambulatorio multicanal de impedancio-pH-metría (MII-pH) y proponen un puntaje para la rumiación que toma en cuenta el número de episodios de reflujo no ácidos por hora, en el periodo postprandial (punto de corte 3 episodios $/ \mathrm{h}=1$ punto) $\mathrm{y}$ el índice sintomático postprandial para la regurgitación (punto de corte $60 \%=1$ punto). Un puntaje de 2 tendría una sensibilidad diagnostica del $91,7 \%$ y una especificidad del 78,6\%. ${ }^{46}$

\section{- Tratamiento}

El enfoque inicial de todo paciente con síndrome de rumiación debe estar dirigido a una explicación detallada de los mecanismos responsables de estos episodios (succión torácica, compresión abdominal y apertura hiatal). Es muy común que estos mecanismos combinados pasen desapercibidos por el paciente dada la breve- dad de estas maniobras.

Las terapias que han demostrados ser efectivas están diseñadas para aumentar farmacológicamente la presión del EEI o restablecer el gradiente de presión esófago-gástrica normal a través de las técnicas conductuales. ${ }^{31,38,39}$

Teóricamente cada subtipo de rumiación debería ser tratado teniendo en cuenta el mecanismo que lo desencadena. De tal manera, la rumiación asociada a los eructos supragástricos debería ser tratada con una terapia cognitiva conductual. En cambio, si la rumiación es secundaria a los episodios de reflujo, el tratamiento debería dirigirse en este sentido. En el caso de la rumiación primaria, la mejor opción terapéutica actualmente es la terapia cognitiva conductual con o sin biorretroalimentación.

\section{- Tratamiento farmacológico}

Baclofeno (agonista de receptores GABA): este fármaco reduce las relajaciones transitorias del EEI y, de esta manera, genera un aumento en la resistencia al paso del contenido gástrico hacia el esófago. En dosis de $10 \mathrm{mg}$ 3 veces por día, comparado con un placebo, ha mostrado un modesto beneficio en la reducción de los eventos de reflujo postprandial (63\% vs. $26 \%) .{ }^{47}$ Los datos sobre su efectividad son limitados y los estudios controlados con un placebo que evidencian este beneficio suelen ser con un número pequeño de pacientes. Futuras investigaciones son necesarias comparando el baclofeno con las intervenciones conductuales.

Neuromoduladores: si bien su uso está ampliamente difundido en el manejo de los desórdenes funcionales digestivos, no existen evidencias hasta el momento de su eficacia en la reducción de los episodios de rumiación. El uso de levosulpirida, antagonista selectivo del receptor D2 de dopamina con actividad procinética, en combinación con psicoterapia ha mostrado un beneficio del $38 \%$ en la mejoría sintomática de estos pacientes. ${ }^{48}$

\section{- Tratamiento quirúrgico}

El uso de la funduplicatura de Nissen ha demostrado ser efectiva en la resolución de los episodios de regurgitación producidos por la ERGE. Existe la descripción de un caso grave de rumiación refractaria con mejoría clínica después de la gastrectomía subtotal. ${ }^{49}$ En ausencia de datos controlados junto con los riesgos de la cirugía, no se recomienda la cirugía como una terapia para el síndrome de rumiación.

\section{- Tratamiento cognitiva conductual}

El abordaje inicial de los pacientes con rumiación podría realizarse con los ejercicios de respiración diafragmática, reservando el tratamiento con biorretroalimentación para la persistencia de los síntomas. 
Las técnicas de respiración diafragmáticas han sido ampliamente difundidas, tanto para el manejo de la rumiación, como para los eructos supragástricos. La modificación de las contracciones abdominales mediante la implementación de respiraciones coordinadas y un mejor control muscular diafragmático puede compensar los episodios de rumiación. Las técnicas de respiración diafragmáticas son fáciles de aprender y pueden ser enseñadas en el consultorio sin necesidad de contar con los equipos de la HRIM o la EMG de la pared abdominal para realizar la biorretroalimentación, los cuales no se encuentran disponibles en todos los centros médicos. ${ }^{31}$

La respiración diafragmática puede ser aprendida fácilmente instruyendo al paciente a colocar una mano sobre el tórax y otra sobre el abdomen. De esta manera, solamente en la inspiración debe moverse la mano abdominal, mientras que la mano del tórax permanece inmóvil. En cada inspiración, se debe producir la protrusión del abdomen sin la movilidad del tórax. Cada inhalación y exhalación debe ser lenta y completa, tratando de completar entre 6 a 8 respiraciones por minuto. Se sugiere una inhalación de 4 segundos y una exhalación de 8 segundos. Estos ejercicios deben practicarse durante 15 minutos después de cada comida. ${ }^{31,38,39}$

Esto puede ser instruido con o sin biorretroalimentación. La biorretroalimentación se refiere al entrenamiento conductual utilizando retroalimentación auditiva o visual por parte del entrenador. En estos casos se utilizan, como mencionamos, la HRIM o la EMG de la pared abdominal. La desventaja de estos métodos son un mayor costo y la baja disponibilidad..$^{40}$

\section{Conclusión}

En esta revisión, se expusieron los principales confundidores de la ERGE haciendo hincapié en el síndrome de rumiación y los eructos supragástricos. Se plantea la necesidad de ampliar el universo diagnóstico ante los pacientes con síntomas típicos de la ERGE que no responden al tratamiento con los IBP y, en especial, en aquellos con regurgitación dominante. Una historia clínica detallada puede orientar al médico tratante, ante la sospecha de dichos desórdenes funcionales, a la derivación oportuna para realizar las pruebas diagnósticas específicas que permitan confirmarlos. Es aquí en donde la HRIM y el monitoreo ambulatorio con impedancio-pH-metría se ofrecen como las mejores herramientas para ayudar a esclarecer cada caso en particular.

\section{Aviso de derechos de autor}

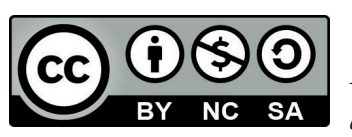

(C) 2021 Acta Gastroenterológica Latinoamericana. Este es un artículo de acceso abierto publicado bajo los términos de la Licencia Creative Commons Attribution (CC BY-NC-SA 4.0), la cual permite el uso, la distribución y la reproducción de forma no comercial, siempre que se cite al autor y la fuente original.

Cite este artículo como: Dulbecco $M$, Vargas Rodríguez $Y$, Guzmán M. Paciente con regurgitación: cómo estudiarla y cuáles son sus principales diagnósticos diferenciales. Acta Gastroenterol Latinoam. 2021;51(1):29-38. https://doi. org/10.52787/jagj 4273

\section{Referencias}

1. Vakil N, van Zanten SV, Kahrilas P, Dent J, Jones R, Global Consensus Group. The Montreal definition and classification of gastroesophageal reflux disease: a global evidence-based consensus. Am J Gastroenterol. 2006;101(8):1900-20.

2. Dent J, El-Serag HB, Wallander MA, Johansson S. Epidemiology of gastro-oesophageal reflux disease: a systematic review. Gut. 2005;54(5):710-7.

3. Chiocca JC, Olmos JA, Salis GB, Soifer LO, Higa R, Marcolongo M; Argentinean Gastro-Oesophageal Reflux Study Group. Prevalence, clinical spectrum and atypical symptoms of gastro-esophageal reflux in Argentina: a nationwide population-based study. Aliment Pharmacol Ther. 2005;22(4):331-42.

4. Klauser AG, Schindlbeck NE, Müller-Lissner SA. Symptoms in gastro-oesophageal reflux disease. Lancet. 1990;335(8683):205-8.

5. Gyawali CP, Kahrilas PJ, Savarino E, Zerbib F, Mion F, Smout AJPM et al. Modern diagnosis of GERD: the Lyon Consensus. Gut. 2018;67(7):1351-62.

6. Olmos JA, Ramos RI, Argonz J, Lozzi RD, Higa R, Gadea O, Perretta CJ, Caro LE, Cerisoli CL, Landoni NA, Salis G. Spectrum of endoscopic findings in patients with symptoms related to gastroesophageal reflux disease (GERD) in Argentina. Nationwide epidemiological study. Gastroenterology. 2009;136(5):A489-90.

7. Numans ME, Lau J, de Wit NJ, Bonis PA. Short-term treatment with proton-pump inhibitors as a test for gastroesophageal reflux disease: a meta-analysis of diagnostic test characteristics. Ann Intern Med. 2004;140(7):518-27.

8. Bravi I, Woodland P, Gill RS, Al-Zinaty M, Bredenoord AJ, Sifrim D. Increased prandial air swallowing and postprandial gas-liquid reflux among patients refractory to proton pump inhibitor therapy. Clin Gastroenterol Hepatol. 2013;11(7):784-9.

9. Herregods TVK, Troelstra M, Weijenborg PW, Bredenoord AJ, Smout AJPM. Patients with refractory reflux symptoms often do not have GERD. Neurogastroenterol Motil. 2015;27(9):1267-73.

10. Katzka DA, Pandolfino JE, Kahrilas PJ. Phenotypes of Gastroesophageal Reflux Disease: Where Rome, Lyon, and Montreal Meet. Clinical Gastroenterology and Hepatology. 2020;18(4):767-76.

11. Gyawali CP, Fass R. Management of Gastroesophageal Reflux Disease. Gastroenterology 2018;154(2):302-18.

12. Moayyedi P, Axon AT. The usefulness of the likelihood ratio in the diagnosis of dyspepsia and gastroesophageal reflux disease. Am J Gastroenterol. 1999;94(11):3122-5.

13. Sawada A, Guzman M, Nikaki K, Sonmez S, Yazaki E, Aziz Q, Woodland P, Rogers B, Gyawali CP, Sifrim D. Identification of Different Phenotypes of Esophageal Reflux Hypersensitivity and Implications for Treatment. Clin Gastroenterol Hepatol. 2020;3565(20):30440-7. 
14. Kahrilas PJ, Howden CW, Hughes N. Response of regurgitation to proton pump inhibitor therapy in clinical trials of gastroesophageal reflux disease. Am J Gastroenterol. 2011;106(8):1419-25.

15. Vaezi MF, Pandolfino JE, Vela MF, Shaheen NJ. White Paper AGA: Optimal Strategies to Define and Diagnose Gastroesophageal Reflux Disease, Clinical Gastroenterology and Hepatology. 2017;15(8):1162-72.

16. Halland M, Pandolfino J, Barba E. Diagnosis and Treatment of Rumination Syndrome. Clin Gastroenterol Hepatol. 2018;16(10):1549-55.

17. Kessing BF, Bredenoord AJ, Smout AJPM. The pathophysiology, diagnosis and treatment of excessive belching symptoms. Am J Gastroenterol. 2014;109(8):1196-203.

18. Sawada A, Fujiwara Y, Sifrim D. Belching in Gastroesophageal Reflux Disease: Literature Review. J Clin Med. 2020;9(10):3360.

19. Westbrook JI, Talley NJ. Empiric clustering of dyspepsia into symptom subgroups: A population-based study. Scand J Gastroenterol. 2002;37(8):917-23.

20. Rey E, Moreno Elola-Olaso C, Rodríguez-Artalejo F, Locke GR, Díaz-Rubio M. Prevalence of atypical symptoms and their association with typical symptoms of gastroesophageal reflux in Spain. Eur J Gastroenterol Hepatol. 2006;18(9):969-75.

21. Kessing BF, Bredenoord AJ, Velosa M, Smout AJPM. Supragastric belches are the main determinants of troublesome belching symptoms in patients with gastro-oesophageal reflux disease. Aliment Pharmacol Ther. 2012;35(9):1073-9.

22. Koukias N, Woodland P, Yazaki E, Sifrim D. Supragastric belching: Prevalence and association with gastroesophageal reflux disease and esophageal hypomotility. J Neurogastroenterol Motil. 2015;21(3):398-403.

23. Saleh CMG, Bredenoord AJ. Utilization of Esophageal Function Testing for the Diagnosis of the Rumination Syndrome and Belching Disorders. Gastrointest Endosc Clin N Am. 2014;24(4):633-42.

24. Stanghellini V, Chan FKL, Hasler WL, Malagelada JR, Suzuki H, Tack J, Talley NJ. Gastroduodenal disorders. Gastroenterology. 2016;150(6):1380-92.

25. Ooi JLS, Vardar R, Sifrim D. Supragastric belching. Curr Opin Gastroenterol. 2016;32(4):302-9.

26. Bredenoord AJ, Weusten BLAM, Timmer R, Smout AJPM. Air swallowing, belching, and reflux in patients with gastroesophageal reflux disease. Am J Gastroenterol. 2006;101(8):1721-6.

27. Bredenoord AJ. Management of Belching, Hiccups, and Aerophagia. Clin Gastroenterol Hepatol. 2013;11(1):6-12.

28. Glasinovic E, Wynter E, Arguero J, Ooi J, Nakagawa K, Yazaki E, et al. Treatment of supragastric belching with cognitive behavioral therapy improves quality of life and reduces acid gastroesophageal reflux. Am J Gastroenterol. 2018;113(4):539-47.

29. Ten Cate L, Herregods TVK, Dejonckere PH, Hemmink GJM, Smout AJPM, Bredenoord AJ. Speech Therapy as Treatment for Supragastric Belching. Dysphagia. 2018;33(5):707-15.

30. Ong AML, Chua LTT, Khor CJL, Asokkumar R, Namasivayam V, Wang YT. Diaphragmatic Breathing Reduces Belching and Proton Pump Inhibitor Refractory Gastroesophageal Reflux Symptoms. Clin Gastroenterol Hepatol. 2018;16(3):407-16.

31. Halland M, Pandolfino J, Barba E. Diagnosis and Treatment of Rumination Syndrome. Clin Gastroenterol Hepatol. 2018;16(10):1549-55.

32. Rommel N, Tack J, Arts J, Caenepeel P, Bisschops R, Sifrim D. Rumination or belching-regurgitation? Differential diagnosis using oesophageal impedance-manometry. Neurogastroenterology \& Motility. 2010;22(4):e97-104.

33. Olmos JA, Pandolfino JE, Piskorz MM, Zamora N, Valdovinos MA, Remes Troche JM et al. Consenso latinoamericano de motilidad esofágica. Acta Gastroenterol Latinoam. 2020;50:8-41.
34. American Psychiatric Association. Feeding and eating disorders. En: Diagnostic and statistical manual of mental disorders. $5 \mathrm{a}$ ed. Washington DC: American Psychiatric Association, 2013.

35. Koloski NA, Talley NJ, Boyce PM. Epidemiology and health care seeking in the functional GI disorders: a population-based study. Am J Gastroenterol. 2002;97(9):2290-9.

36. López-Colombo A, Morgan D, Bravo-González D, Montiel-Jarquín A, Méndez-Martínez S, Schmulson M. The epidemiology of functional gastrointestinal disorders in Mexico: a population-based study. Gastroenterol Res Pract. 2012;2012:606174.

37. Yadlapati R, Tye M, Roman S, Kahrilas PJ, Ritter K, Pandolfino JE. Postprandial high-resolution impedance manometry identifies mechanisms of nonresponse to proton pump inhibitors. Clin Gastroenterol Hepatol. 2018;16(2):211-8.

38. Vachhani H, De Souza Ribeiro B, Schey R. Rumination Syndrome: Recognition and Treatment. Curr Treat Options Gastroenterol. 2020;18:60-8.

39. Absah I, Rishi A, Talley NJ, Katzka D, Halland M. Rumination syndrome: Pathophysiology, diagnosis, and treatment. Neurogastroenterol Motil. 2017;29(4):e12954.

40. Barba E, Accarino A, Soldevilla A, Malagelada JR, Azpiroz F. Randomized, placebo-controlled trial of biofeedback for the treatment of rumination. Am J Gastroenterol. 2016;111(7):1007-13.

41. Thumshirn M, Camilleri M, Hanson RB, Williams DE, Schei AJ, Kammer PP. Gastric mechanosensory and lower esophageal sphincter function in rumination syndrome. Am J Physiol. 1998;275(2):G314-21.

42. Bredenoord AJ, Chial HJ, Camilleri M, Mullan BP, Murray JA. Gastric accommodation and emptying in evaluation of patients with upper gastrointestinal symptoms. Clin Gastroenterol Hepatol. 2003;1(4):264-72.

43. Wang X, Luscombe GM, Boyd C, Kellow J, Abraham S. Functional gastrointestinal disorders in eating disorder patients: altered distribution and predictors using ROME III compared to ROME II criteria. World J Gastroenterol. 2014;20(43):16293-9.

44. Kessing BF, Bredenoord AJ, Smout AJPM. Objective manometric criteria for the rumination syndrome. Am J Gastroenterol. 2014;109:52-9.

45. Yadlapati R, Kharilas PJ, Fox MR, Bredenoord AJ, Gyawali CP, Roman $\mathrm{S}$ et al. Esophageal motility disorders on high-resolution manometry: Chicago classification version 4.0. Neurogastroenterology \& Motility. 2020;33:e14058.

46. Nakagawa K, Sawada A, Hoshikawa Y, Nikaki K, Sonmez S, Woodland P, et al. Persistent postprandial regurgitation vs rumination in patients with refractory gastroesophageal reflux disease symptoms: identification of a distinct rumination pattern using ambulatory impedance-pH monitoring. Am J Gastroenterol. 2019;114(8):1248-55.

47. Blondeau K, Boecxstaens V, Rommel N, Farré R, Depeyper S, Holvoet L, Boeckxstaens G, Tack JF. Baclofen improves symptoms and reduces postprandial flow events in patients with rumination and supragastric belching. Clin Gastroenterol Hepatol. 2012;10(4):379-84.

48. Lee H, Rhee PL, Park EH, Kim JH, Son HJ, Kim JJ, Rhee JC. Clinical outcome of rumination syndrome in adults without psychiatric illness: a prospective study. J Gastroenterol Hepatol. 2007;22(11):1741-7.

49. Cooper CJ, Otoukesh S, Mojtahedzadeh M, Galvis JM, McCallum RW. Subtotal Gastrectomy as "Last Resort" Consideration in the Management of Refractory Rumination Syndrome. Gastroenterology Res. 2014;7(3-4):98-101. 